\title{
PERFORMANCE OF 10- AND 20-TARGET MSE CLASSIFIERS ${ }^{1}$
}

\author{
Leslie M. Novak, Gregory J. Owirka, and William S. Brower \\ Lincoln Laboratory Massachusetts Institute of Technology \\ 244 Wood Street \\ Lexington, MA 02420-9185
}

\begin{abstract}
MIT Lincoln Laboratory is responsible for developing the ATR (automatic target recognition) system for the DARPA-sponsored SAIP program; the baseline ATR system recognizes 10 GOB (ground order of battle) targets; the enhanced version of SAIP requires the ATR system to recognize 20 GOB targets. This paper presents ATR performance results for 10- and 20-target MSE classifiers using highresolution SAR (synthetic aperture radar) imagery.
\end{abstract}

Keywords: automatic target recognition, synthetic aperture radar, mean square error classifier, tactical targets, stationary targets

\section{INTRODUCTION}

MIT Lincoln Laboratory is responsible for developing the ATR system for the DARPA-sponsored SAIP program [1,2]. SAIP supports new sensor platforms such as the Global Hawk system [3,4] which gathers wide area SAR stripmap imagery at medium resolution $(1.0 \mathrm{~m} \times 1.0 \mathrm{~m})$ and SAR spotlight imagery at high resolution $(0.3 \mathrm{~m} \times 0.3 \mathrm{~m})$. The classification stage of the SAIP ATR provides target recognition at both medium and high resolution. In high resolution spotlight mode, conventional 2DFFT image formation processing is used to construct the $0.3 \mathrm{~m} \times 0.3 \mathrm{~m}$ resolution SAR imagery. In medium resolution stripmap mode, a superresolution image formation algorithm is used to enhance SAR image resolution. This new image formation algorithm enhances the resolution of the $1.0 \mathrm{~m} \times 1.0 \mathrm{~m}$

\footnotetext{
${ }^{1}$ This work was sponsored by the Defense Advanced Research Projects Agency under Air Force Contract F19628-95-C-0002. Opinions, interpretations, conclusions, and recommendations are those of the authors and are not necessarily endorsed by the United States Government.
} 
imagery to approximately $0.5 \mathrm{~m} \times 0.5 \mathrm{~m}$. Reference [5] focuses on ATR performance of the SAIP MSE classifier using medium resolution $(1.0 \mathrm{~m} \times 1.0 \mathrm{~m})$ SAR imagery; this paper focuses on a comparison of ATR performance for 10- and 20-target MSE classifiers using high resolution $(0.3 \mathrm{~m} \times 0.3 \mathrm{~m}) \mathrm{SAR}$ imagery.

A brief overview of the paper follows. Section 2 discusses some initial classifier studes and describes the SAR imagery used in these studies; photographs and high resolution SAR images of the ground order of battle (GOB) targets are shown. Section 3 describes classifier training and testing, and presents evaluations of the performance of the 10- and 20-target MSE classifiers. In Section 4 we consider the effects of severe target configuration variabilities (extended operating conditions), including classifier testing using images of targets placed in revetments, and images of an M109 target with severe turret rotations (turret rotated $90 \mathrm{deg}$ from forward position). Finally, Section 5 summarizes the important results from these studies.

\section{BACKGROUND AND DATA DESCRIPTION}

The synthetic aperture radar imagery used in these studies was provided to Lincoln Laboratory by Wright Laboratories, WPAFB, Dayton, Ohio. The data were gathered by the Sandia X-band, HHpolarization SAR sensor at two different sites in support of the DARPA-sponsored MSTAR program [6]. The first MSTAR collection (MSTAR \#1) took place in fall 1995 at Redstone Arsenal, Huntsville, Alabama; the second MSTAR collection (MSTAR \#2) took place in fall 1996 at Eglin AFB, Ft. Walton Beach, Florida. In each collection, a large number of military targets were imaged in spotlight mode, over $360 \mathrm{deg}$ of target aspect, and at $0.3 \mathrm{~m} \times 0.3 \mathrm{~m}$ resolution.

Our initial studies [1,2] evaluated the performance and summarized the results of a 10-target MSE classifier using imagery of the 18 distinct targets contained in the MSTAR \#1 data set. Figure 1 shows a typical SAR spotlight image $(0.3 \mathrm{~m} \times 0.3 \mathrm{~m}$ resolution) of the Redstone Arsenal target array. We used 15 deg depression target images to construct a 10-target classifier. The classifier was "trained" by constructing classifier templates using SAR images of the following targets: BMP2\#1, M2\#1, T72\#1, BTR60, BTR70, M1, M109, M110, M113, and M548 (nomenclature explained in Figure 2). The target array shown in Figure 1 includes three versions each of the BMP2 armored personnel carrier, the M2 
infantry fighting vehicle, and the T72 main battle tank. The three T72 tanks varied significantly from tank to tank; T72\#1 used in training the classifier had skirts along both sides of the target; T72\#2 had fuel drums (barrels) mounted on the rear of the tank; T72\#3 had neither skirts nor barrels. The classifier was tested using the remaining 8 targets that were not used in training the classifier: two BMP2s, two M2s, two T72s, the HMMWV, and the M35. In our initial studies, the HMMWV and the M35 were used as confuser vehicles (i.e., vehicles not included in the set of 10 targets that the classifier was trained to recognize); the other 6 test targets provided independent classifier testing data (data not used in classifier training). One important conclusion gleaned from these initial studies was that the ability to correctly classify the independent T72 targets depended strongly on how closely the target configuration matched that of the tank used in training the classifier. Because of the presence of the fuel drums located on the rear of the tank, T72\#2 was called unknown a significant number of times. Using additional T72 tank data from MSTAR \#2 data set, we demonstrate in this paper that intraclass variability is a very important issue for classifier design.

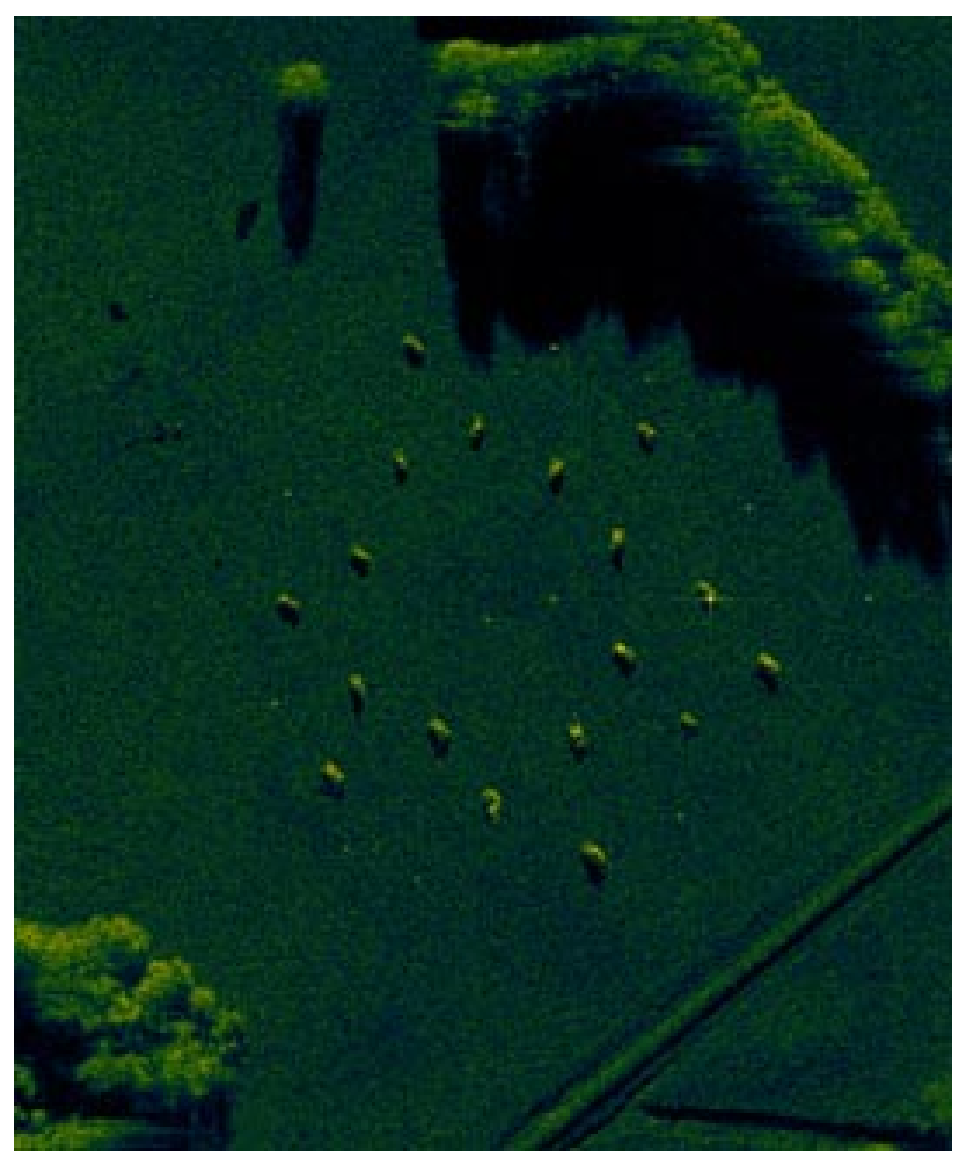

Figure 1. Typical SAR spotlight image $(0.3 \mathrm{~m} \times 0.3 \mathrm{~m}$ resolution) of the Redstone Arsenal target array. 
This paper compares the performance of the 10-target classifier with the performance obtained from the newly implemented 20-target MSE classifier. To implement the 20-target MSE classifier we combined 11 target types imaged during the MSTAR \#1 collection with 9 target types imaged during the MSTAR \#2 collection ( both data sets at 15 -deg depression). Figure 3 shows a typical SAR spotlight image (0.3 $\mathrm{m} \times 0.3 \mathrm{~m}$ resolution) of the Eglin AFB target array. Photographs of the targets used to implement the 20-target MSE classifier are shown in Figure 4.

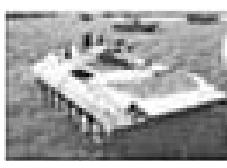

BMP2 \#1

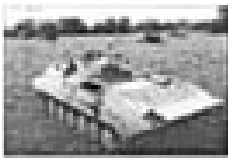

BMP2 \#2

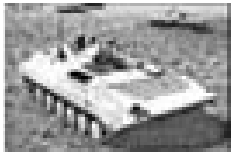

BMP2 \#3

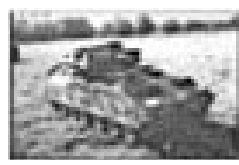

M2 \#1

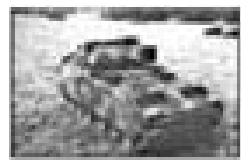

M2 \#2

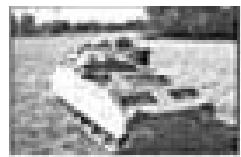

M2 \#3

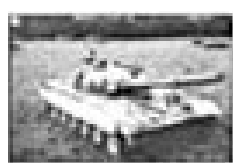

T72 \#1

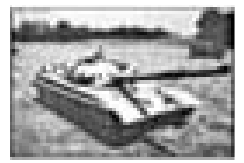

T72 \#2

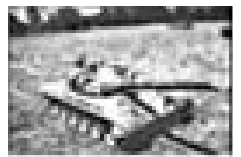

T72 \#3

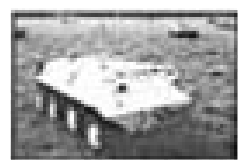

BTR60

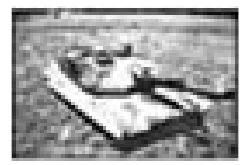

M1

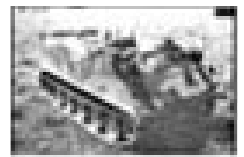

M113

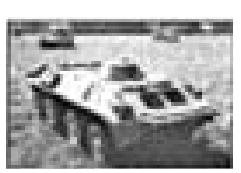

BTR70

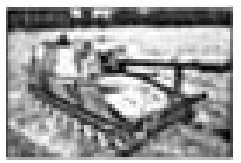

M109

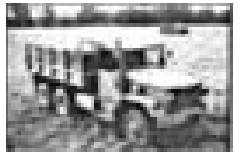

M35

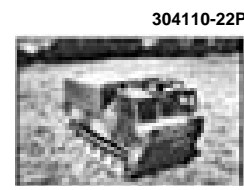

M548

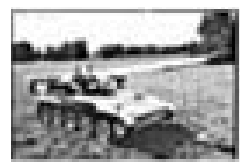

M110

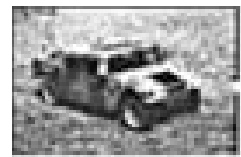

HMMWV

Figure 2. Photographs of the 18 targets from the MSTAR \#1 collection. The classifier was trained with 10 targets (BMP2\#1, M2\#1, T72\#1, BTR60, BTR70, M548, M1, M109, M110, M113). Six independent targets (BMP2\#2, M2\#2, T72\#2, BMP2\#3, M2\#3, T72\#3) and two confuser targets (M35, HMMWV) provided test data for the classifier. 


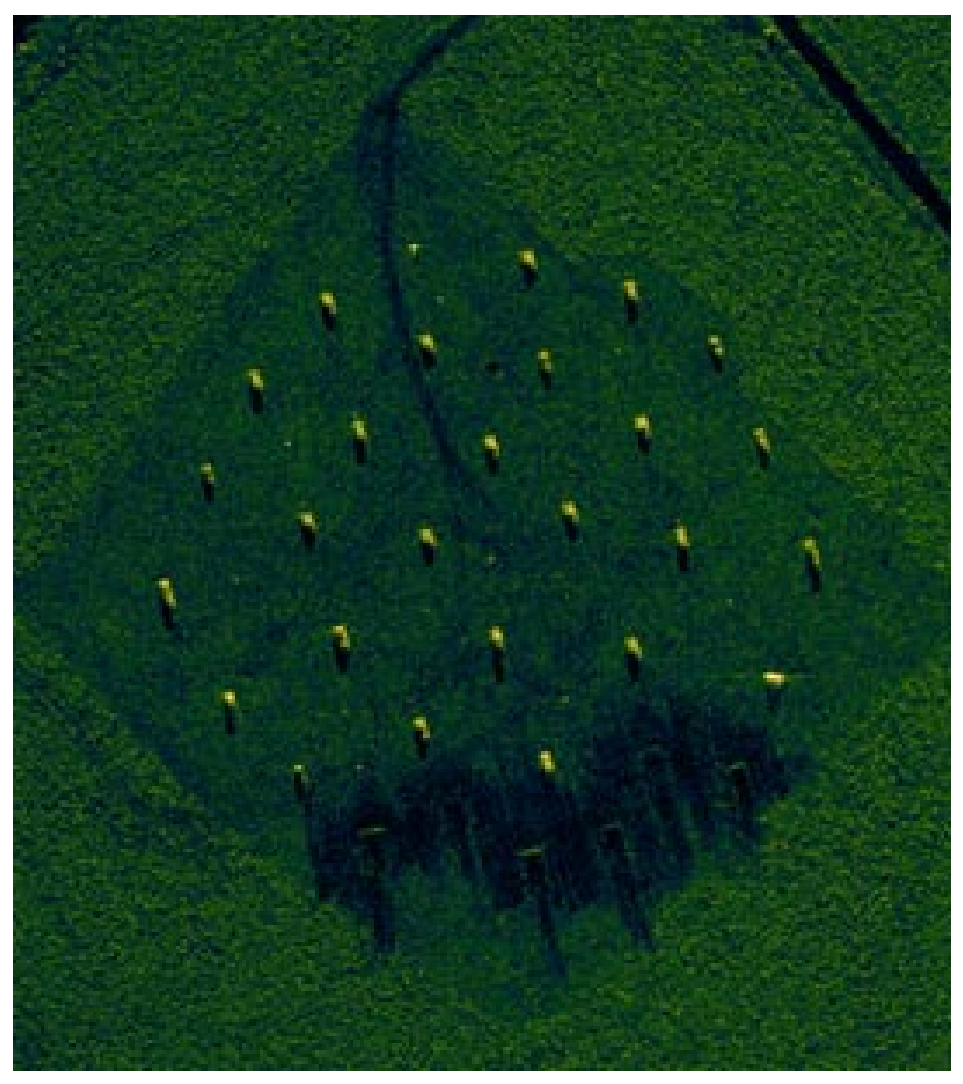

Figure 3. Typical SAR spotlight image (0.3 $\mathrm{m} \times 0.3 \mathrm{~m}$ resolution) of the Eglin AFB target array. 


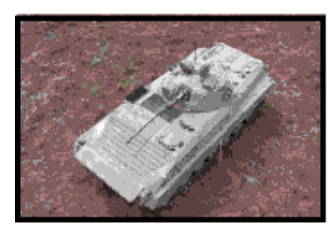

BMP2

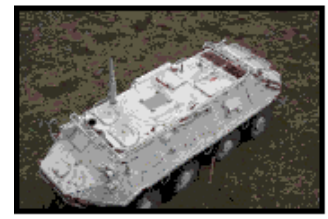

BTR60

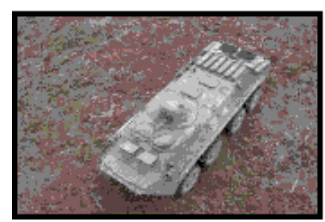

BTR70

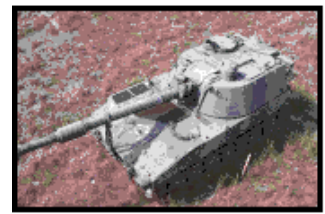

M109

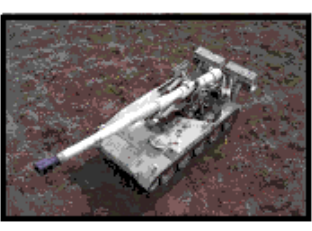

M-10

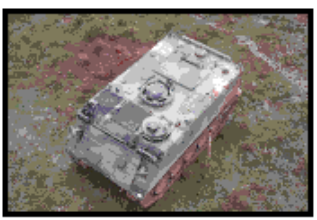

$M+13$

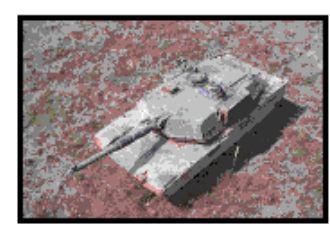

M-1

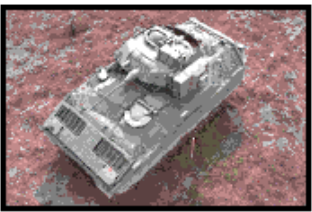

M2

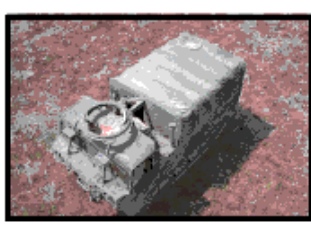

M548

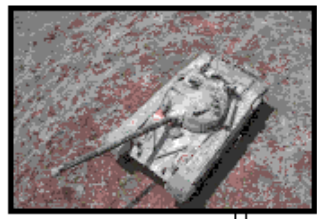

T72

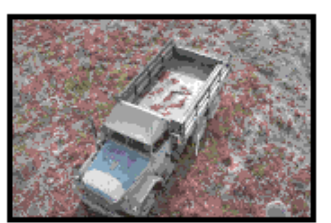

M35

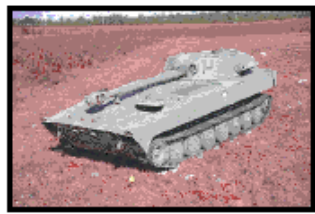

251

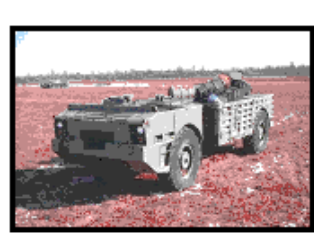

M520

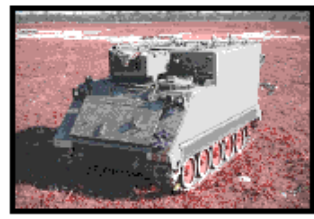

M577

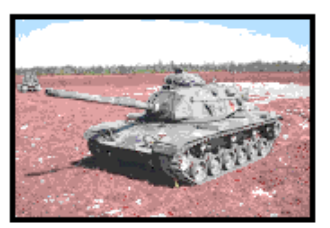

M60

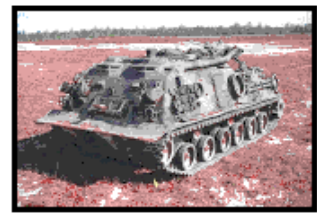

M88

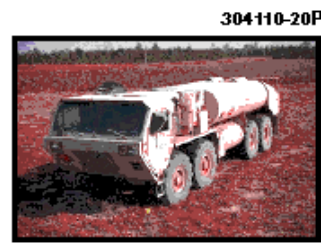

M978

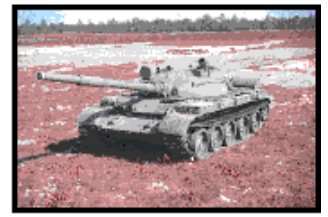

T62

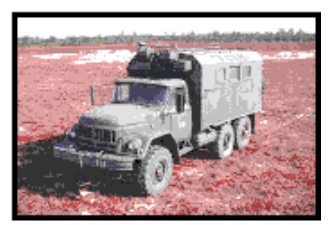

ZIL

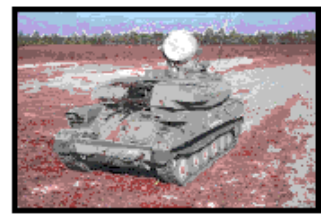

ZSU

Figure 4. Photographs of the 20 targets from the MSTAR \#1 and MSTAR \#2 collections used to train the 20-target classifier.

\section{PERFORMANCE RESULTS}

This section of the paper summarizes the ATR performance achieved by the MSE classifier using $0.3 \mathrm{~m}$ $\times 0.3 \mathrm{~m}$ resolution SAR imagery. The 10-target and 20-target classifiers were implemented by constructing 72 templates per target. These templates were obtained by generating target images every degree in aspect around the target. Five consecutive images were then averaged to form 72 average images per target. The templates were then obtained by isolating the clutter-free target pixels from each average image, providing 72 templates spanning a total 360-deg aspect coverage per target. Both classifiers were initially tested using the 6 independent targets from the MSTAR \#1 collection. The results of these evaluations are summarized in Table 1, which presents the classifier confusion matrices for the 10-target classifier trained using MSTAR \#1 data and tested on the 6 MSTAR \#1 independent 
test targets (top) and for the 20-target classifier trained using MSTAR \#1 and MSTAR \#2 data and tested on 6 MSTAR \#1 independent test targets (bottom).

Table 1

Confusion Matrices for the 10-and 20 Target Classifiers Using 0.3 M ×0.3 M Resolution Imagery

(Six Independent Targets From the MSTAR \#1 Data Set)

Number of Targets Classified As

304110-19B

\begin{tabular}{|c|c|c|c|c|c|c|c|c|c|c|c|}
\cline { 3 - 12 } \multicolumn{1}{c|}{} & BMP2 & BTR60 & BTR70 & M109 & M110 & M113 & M1 & M2 & M548 & T72 & Unk \\
\hline BMP2 \#2 & 187 & & 1 & 1 & & & & 2 & & 1 & 4 \\
\hline BMP2 \#3 & 194 & & & 1 & & & & 1 & & & \\
\hline M2 \#2 & & & & & & & & 196 & & & \\
\hline M2 \#3 & & & & & & 1 & & 194 & & & 1 \\
\hline T72 \#2 & & 1 & & & & & & & & 158 & 36 \\
\hline T72 \#3 & & & & & & & & & & 186 & 5 \\
\hline
\end{tabular}

10-Target Classifier Pcc $=95.3 \%$

Number of Targets Classified As

20-Target Classifier Pcc $=94.0 \%$

\begin{tabular}{|c|c|c|c|c|c|c|c|c|c|c|c|c|c|c|c|c|c|c|c|c|c|}
\hline & BMP2 & BTR60 & \begin{tabular}{|l|} 
BTR70 \\
\end{tabular} & M109 & M110 & M113 & M1 & M2 & M548 & T72 & M35 & $2 S 1$ & M520 & M577 & M60 & M88 & M978 & T62 & ZIL & zsu & Unk \\
\hline BMP2 \#2 & 186 & & 1 & 1 & & & & 2 & & 1 & & & & 1 & & & & & & & 4 \\
\hline BMP2 \#3 & 193 & & & & & & & 1 & & & & & & 2 & & & & & & & \\
\hline M2 \#2 & & & & & & & & 196 & & & & & & & & & & & & & \\
\hline M2 \#3 & & & & & & 1 & & 193 & & & & & & 1 & & & & & & & 1 \\
\hline T72 \#2 & & 1 & & & & & & & & 146 & & & & 2 & 2 & & & 9 & & & 35 \\
\hline T72 \#3 & & & & & & & & & & 186 & & & & & & & & & & & 5 \\
\hline
\end{tabular}

When the 10-target classifier was tested using the independent MSTAR \#1 test data, nearly perfect performance was achieved. Probability of correct classification is $95.3 \%$ against the 6 independent targets. Note, however, that the performance for the T72 tank with fuel drums on the rear (T72\#2) was somewhat reduced; 36 images of the 195 total were declared unknown by the classifier. When the 20target classifier was tested using the same independent MSTAR \#1 test data, the probability of correct classification degraded slightly to $94.0 \%$. The number of targets declared unknown by the 20-target classifier was approximately the same as for the 10-target classifier (45 images of the total 1170). 
Both classifiers were then tested using independent test data (three BTR70s and four M109s) in controlled configurations from the MSTAR \#2 collection. Table 2 presents the classifier confusion matrices for the original 10-target classifier tested on the 7 MSTAR \#2 independent test targets (top) and for the 20-target classifier tested on the 7 MSTAR \#2 independent test targets (bottom). The probabilities of correct classification against these independent test data are $96.8 \%$ and $93.1 \%$ for the 10 and 20-target classifiers, respectively. This test illustrates that classifier templates developed from the MSTAR \#1 collection work equally well when tested against these independent test target images from the MSTAR \#2 collection. Note that for the 20-target classifier, a small number of BTR70s were incorrectly classified as 2S1s (26 images of the total 821) and a small number of M109s were incorrectly classified as M577s (33 images of the total 1095).

Table 2

Confusion Matrices for the 10- and 20-Target Classifiers

(Seven Independent Targets From the MSTAR \#2 Data Set)

304110-18B

Number of Targets Classified As

\begin{tabular}{|c|c|c|c|c|c|c|c|c|c|c|c|}
\cline { 2 - 11 } \multicolumn{1}{c|}{} & BMP2 & BTR60 & BTR70 & M109 & M110 & M113 & M1 & M2 & M548 & T72 & Unk \\
\hline BTR70 \#1 & & & 270 & & & & & & & & 4 \\
\hline BTR70 \#2 & & & 260 & & & & & & & & 13 \\
\hline BTR70 \#3 & & & 252 & & & & & & & & 22 \\
\hline M109 \#1 & & & & 271 & & & & & & & 2 \\
\hline M109 \#2 & 1 & & & 260 & & & & & & & 13 \\
\hline M109 \#3 & & & & 273 & & & & & & & 1 \\
\hline M109 \#4 & & & & 268 & & & & 3 & & & 3 \\
\hline
\end{tabular}

10-Target Classifier Pcc $=96.8 \%$

Number of Targets Classified As

20-Target Classifier Pcc $=93.1 \%$

\begin{tabular}{|c|c|c|c|c|c|c|c|c|c|c|c|c|c|c|c|c|c|c|c|c|c|}
\cline { 2 - 5 } & BMP2 & BTR60 & BTR70 & M109 & M110 & M113 & M1 & M2 & M548 & T72 & M35 & 2S1 & M520 & M577 & M60 & M88 & M978 & T62 & ZIL & ZSU & Unk \\
\hline BTR70 \#1 & & & 267 & & & & & & & & & & & 1 & & 1 & & & 1 & & 4 \\
\hline BTR70 \#2 & & & 249 & & & & & & & & & 10 & & 1 & & & & 1 & & & 12 \\
\hline BTR70 \#3 & & 235 & & & & & & & & & 16 & & & & 1 & & 1 & 3 & & 18 \\
\hline M109 \#1 & & & 266 & & & & & & & & & & 4 & & & & 1 & & & 2 \\
\hline M109 \#2 & 1 & & & 243 & & & & & & & & & & 15 & & & & & 4 & 1 & 10 \\
\hline M109 \#3 & & & & 268 & & & & & & & & & & 4 & & & & 1 & & & 1 \\
\hline M109 \#4 & & & & 255 & & & & 3 & & & & & & 10 & & & & & 4 & & 2 \\
\hline
\end{tabular}


The MSTAR \#2 collection imaged eight different-serial-numbered T72 tanks in a variety of configurations, as described in Table 3. We tested the 10- and 20-target classifiers using target images of seven of the independent T72 tanks from the MSTAR \#2 collection. Note that a single T72 tank from the MSTAR \#1 collection was used to train both classifiers; its configuration was skirts/no-barrels (S/NB; i.e., skirts along both sides of the tank but no fuel drums mounted at the rear).

Table 3

Intraclass Variability Matrix

(Eight T72 Tanks From the MSTAR \#2 Data Set)

T72 Intraclass Variability Matrix

$332022-1$

\begin{tabular}{|c|c|}
\hline Notation & Configuration of Target \\
\hline S/B & Skirts/barrels (fuel drums) \\
\hline S/NB & Skirts/no barrels \\
\hline NS/B & No skirts/barrels \\
\hline NS/NB & No skirts/no barrels \\
\hline S/B/A & Skirts/barrels/reactive armor \\
\hline
\end{tabular}

As shown in Table 4, the 10-target classifier rejected a large number of T72 tank images (408 of the total 1918), declaring them unknown. The confusion matrix indicates that 100 images of T72\#7 were rejected, 104 images of T72\#6 were rejected, and 82 images of T72\#5 were rejected. We investigated the various T72 configurations more carefully. For example, Figures 5 and 6 are photographs of the tank with and without the reactive armor; this variability is expected to cause a large increase in the mean-square-error calculated by the classifier. Figure 7 shows MSE histograms for two targets. One is a T72 in the S/NB configuration; since the T72 used to train the classifier had the same configuration, the majority of test inputs are below the MSE baseline threshold of 55. Figure 7 also shows the MSE histogram for a T72 with the skirts/barrels/reactive-armor configuration; the curve indicates a large fraction of these scores is above the threshold of 55, and these test inputs are rejected as unknown by the classifier. 
The 20-target-classifier confusion matrix presented in Table 4 illustrates that only 1130 test images were correctly classified of the total 1918; also, 303 test images were declared unknown. Increasing the number of target classes from 10 to 20 resulted in many T72 test images being declared T62s or M60s in the 20-target; this happened because the T72 target training was done using only the S/NB configuration target data from the MSTAR \#1 collection.

Table 4

Confusion Matrices for the 10-AND 20-Target Classifiers

(Seven Tanks From the MSTAR \#2 Data Set)

Number of Targets Classified As

\begin{tabular}{|c|c|c|c|c|c|c|c|c|c|c|c|c|}
\hline & & BMP2 & BTR60 & BTR70 & M109 & M110 & M113 & M1 & M2 & M548 & T72 & Unk \\
\hline \multirow{3}{*}{$\mathrm{S} / \mathrm{NB}$} & $\mathrm{T} 72 \# 1$ & & & & 1 & 1 & & 1 & 9 & & 234 & 28 \\
\hline & $\mathrm{T} 72 \# 2$ & 1 & & & & & & 4 & 1 & & 249 & 19 \\
\hline & $\mathrm{T} 72 \# 3$ & & & & & & & 3 & 5 & & 234 & 29 \\
\hline $\mathrm{NS} / \mathrm{NB}$ & $\mathrm{T} 72 \# 4$ & 9 & & 1 & & 1 & & 3 & 3 & & 211 & 46 \\
\hline$S / B$ & $\mathrm{T72} \# 5$ & 3 & 1 & & & & 1 & 1 & 2 & & 184 & 82 \\
\hline $\mathrm{NS} / \mathrm{B}$ & T72 \#6 & 9 & & & 1 & & 4 & & 3 & & 153 & 104 \\
\hline $\mathbf{S} / \mathbf{B} / \mathbf{A}$ & T72 \#7 & 10 & & & & 1 & & 7 & 3 & & 153 & 100 \\
\hline
\end{tabular}

10-Target Classifier Pcc $=74.0 \%$

Number of Targets Classified As

20-Target Classifier Pcc $=59.0 \%$

\begin{tabular}{|c|c|c|c|c|c|c|c|c|c|c|c|c|c|c|c|c|c|c|c|c|c|c|}
\hline & & BMP2 & BTR60 & BTR70 & M109 & M110 & M113 & M1 & M2 & M548 & T72 & M35 & $2 \mathrm{~S} 1$ & M520 & M577 & M60 & M88 & M978 & T62 & ZIL & zsU & Unk \\
\hline \multirow{3}{*}{$\mathrm{S} / \mathrm{NB}$} & $\mathrm{T} 72 \# 1$ & & & & & 1 & & & 8 & & 200 & & & & 8 & 22 & & & 10 & & & 22 \\
\hline & T72 \#2 & & & & & & & 2 & 1 & & 232 & & & & 6 & 13 & & & 6 & & & 14 \\
\hline & $\mathrm{T} 72 \# 3$ & & & & & & & 2 & 5 & & 206 & & & & 1 & 24 & 1 & & 7 & 1 & & 24 \\
\hline $\mathrm{NS} / \mathrm{NB}$ & $\mid \mathrm{T} 72 \# 4$ & 4 & & 1 & & & & 3 & 1 & & 161 & & 2 & & 4 & 31 & & & 31 & 1 & & 35 \\
\hline$S / B$ & T72 \#5 & & 1 & & & & 1 & 1 & 1 & & 142 & & 2 & & 6 & 8 & & & 45 & 1 & & 66 \\
\hline $\mathbf{N S} / \mathbf{B}$ & T72 \#6 & 6 & & & & & 3 & & 1 & & 88 & & 1 & & 6 & 12 & & & 90 & & 1 & 66 \\
\hline$S / B / A$ & T72 \#7 & 5 & & & & & & 5 & & & 101 & & 2 & & 22 & 30 & & & 32 & 1 & & 76 \\
\hline
\end{tabular}




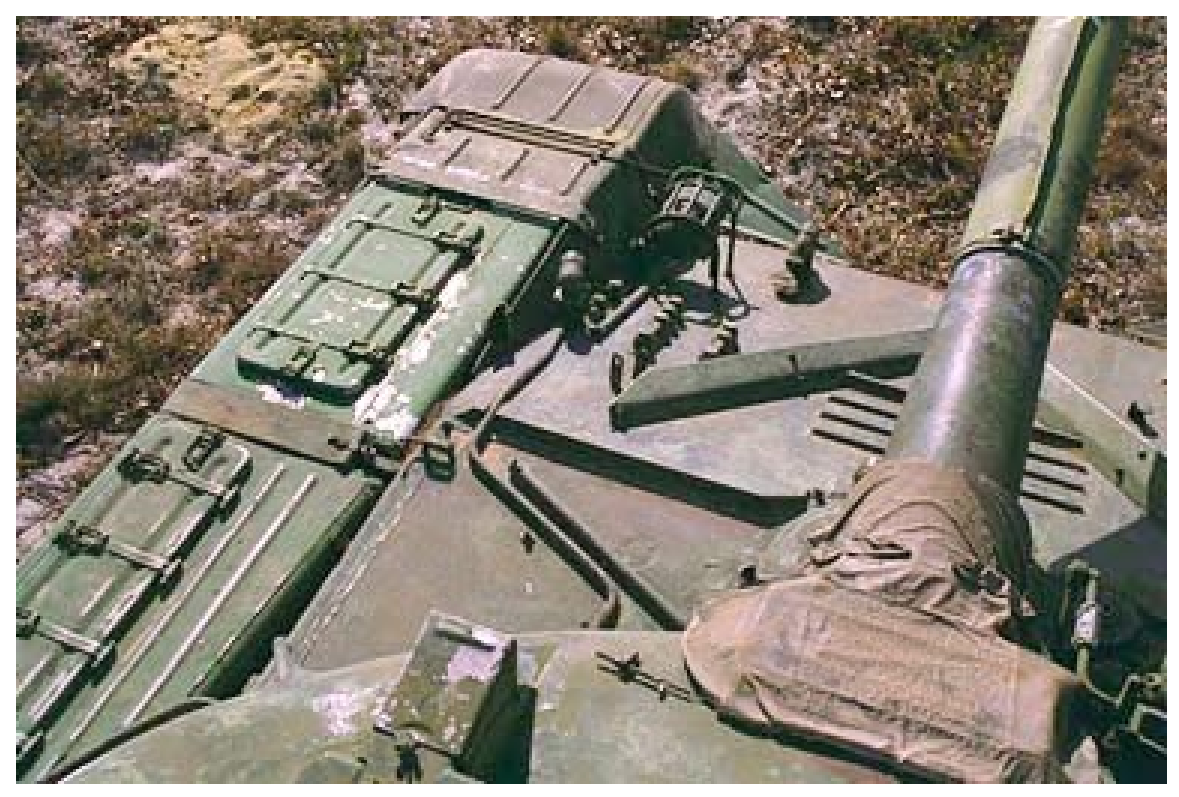

Figure 5. Top view of a typical T72 tank without reactive armor.

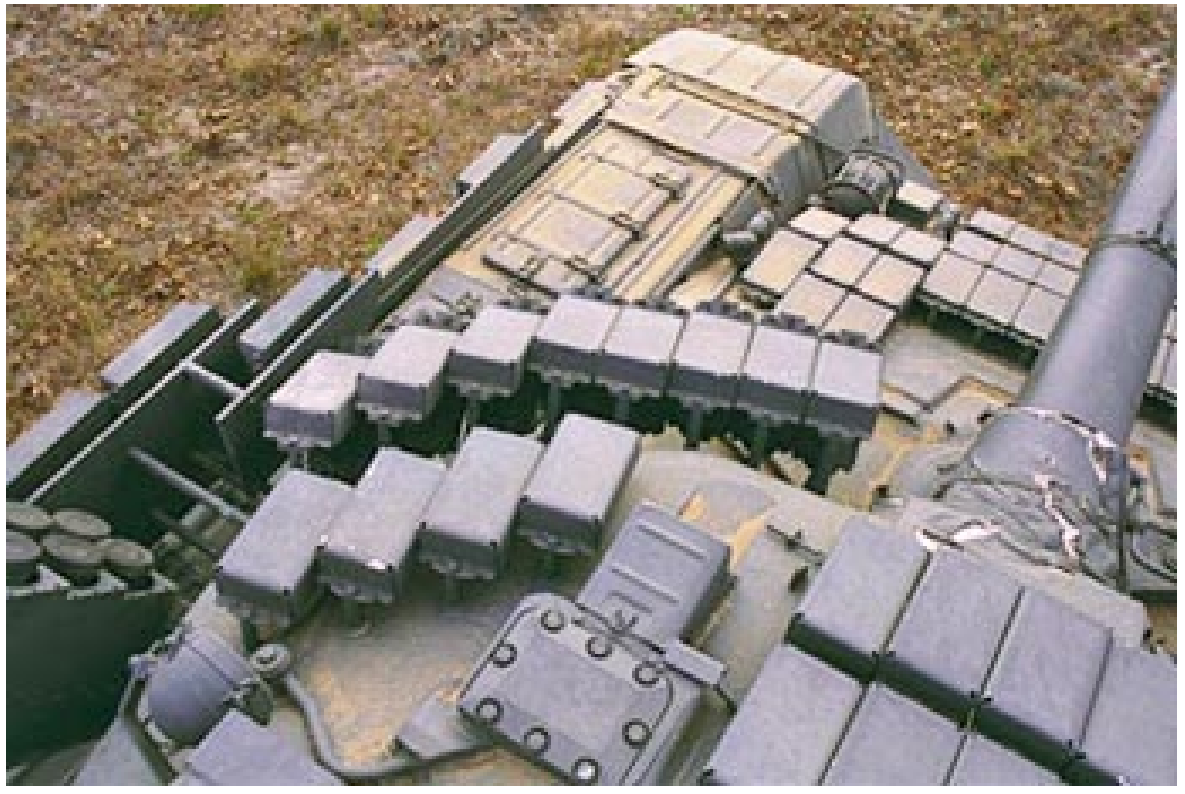

Figure 6. Top view of a T72 tank with reactive armor covering a significant portion of the tank surface. 


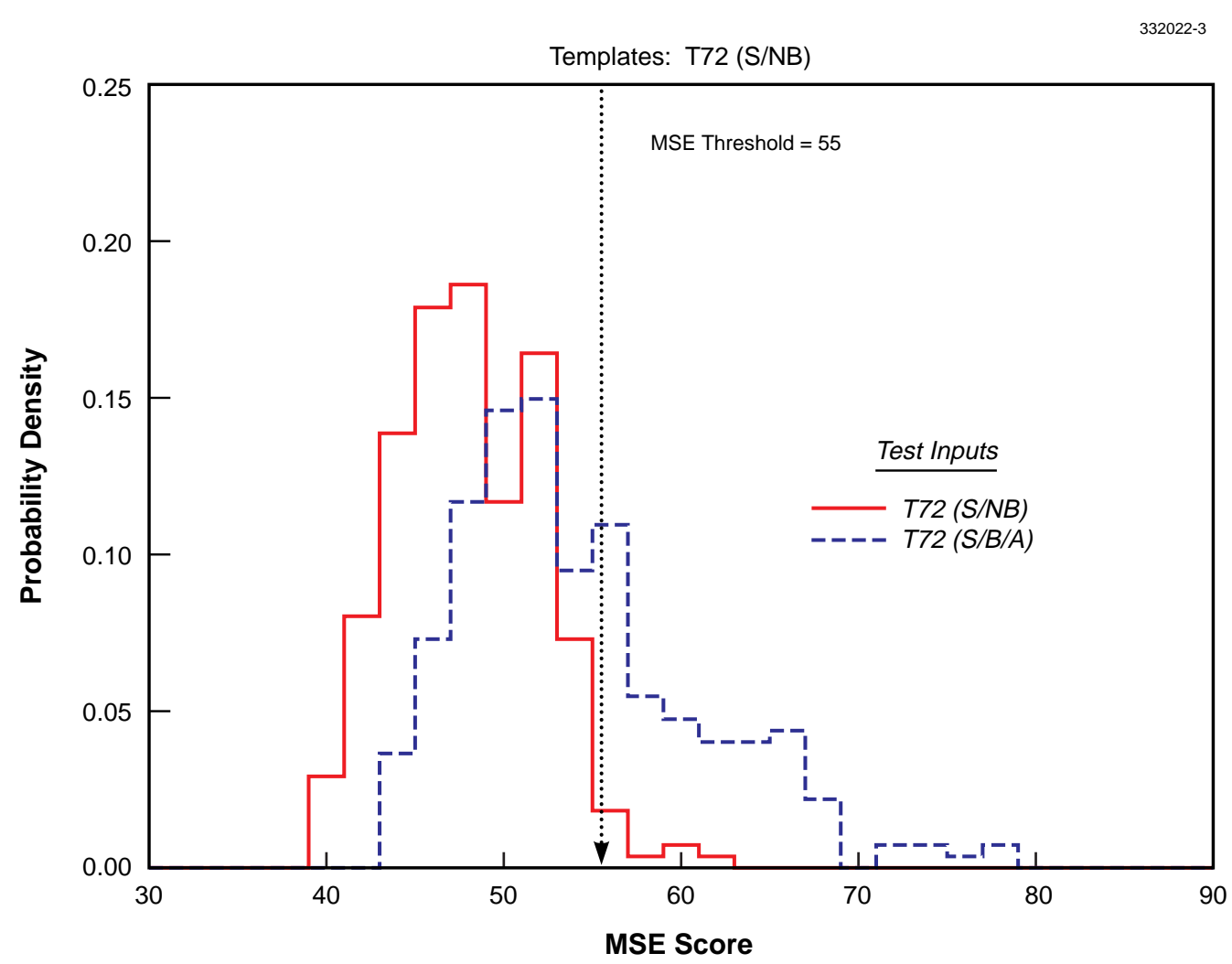

Figure 7. MSE score distributions of two independent T72 test inputs against the T72 templates used to train the classifier. The solid line is the distribution of scores from a tank with the same configuration as the training tank (skirts, no barrels). The dashed line is the distribution of scores from a tank configured with skirts, barrels, and reactive armor. A large fraction of the latter scores are above the baseline MSE classifier threshold (dotted line) and are thus rejected as unknown.

Figure 8 compares T72 test images (center) with the T72 training images from MSTAR \#1 (left) and the T62 target images from MSTAR \#2 (right). The center T72 test images appear visually more similar to the T62 target images because the configuration for each of these targets is no-skirts/barrels (NS/B; see Figure 4). These observations prompted an experimental classifier augmentation, leading to the confusion matrices presented in Table 5. We determined that by augmenting the classifier template sets with an additional template set for a T72 tank having an NS/B configuration, the probabilities of correct classification improved to $93.9 \%$ and $90.4 \%$ for the 10 - and the 20 -target classifiers, respectively. (Although we have added additional T72 templates to each of the classifiers, we still denote these classifiers as 10- and 20-target classifiers because they only identify 10 and 20 unique target types). The 10- and 20-target classifiers discussed earlier (Tables 1, 2, 4) used 740 templates and 1440 templates, 
respectively; the 10- and 20-target classifiers presented in Table 5 used 812 templates and 1512 templates, respectively.

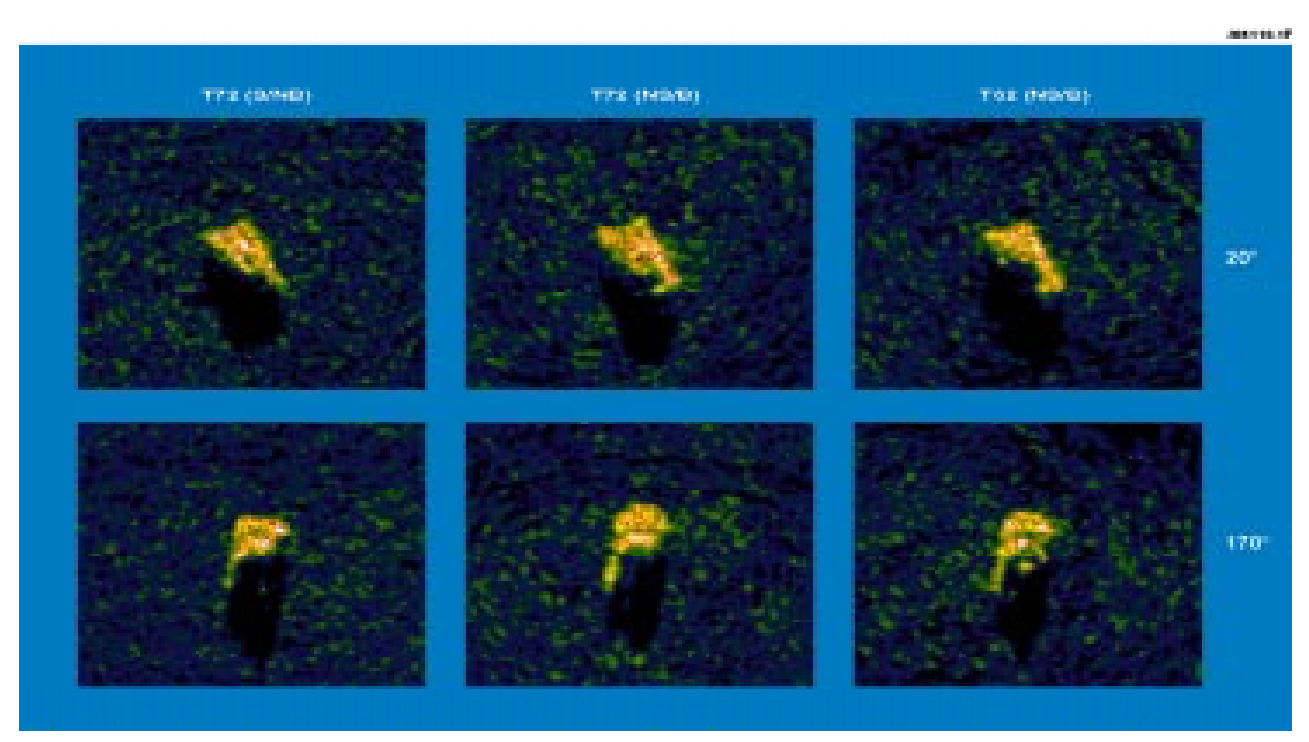

Figure 8. SAR images of a T72 with skirts and without barrels (left column), a T72 without skirts and with barrels (center column), and a T62 without skirts and with barrels (right column) at two aspect angles $20 \mathrm{deg}$ (top row) and $170 \mathrm{deg}$ (bottom row). The T72 on the left and the T62 on the right are used to train the 20-target classifier while the center T72 provides independent test data. The center T72 is visually more similar to the identically configured T62 than the differently configured T72, thus prompting the addition of additional T72 templates to mitigate the T72 intraclass variability. 
Table 5

Confusion Matrices for the 10- and 20-Target Classifiers (with additional T72 templates)

(Seven Tanks From the MSTAR \#2 Data Set)

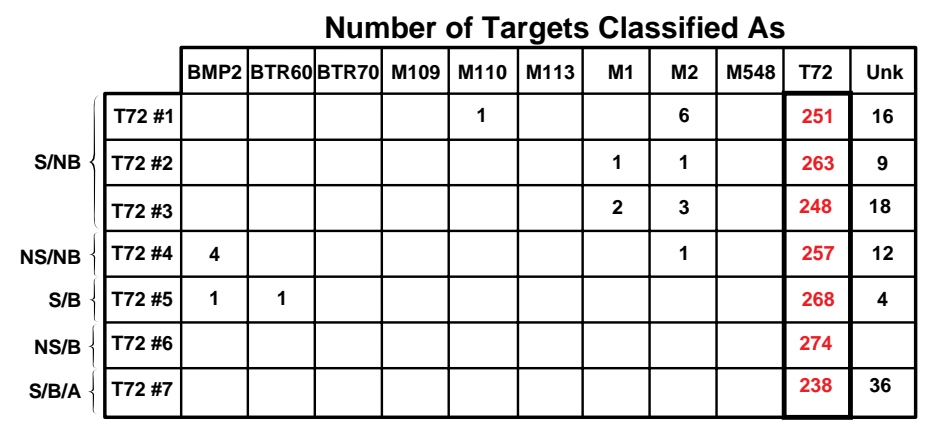

10-Target Classifier Pcc $=93.9 \%$

20-Target Classifier Pcc $=90.4 \%$

Number of Targets Classified As

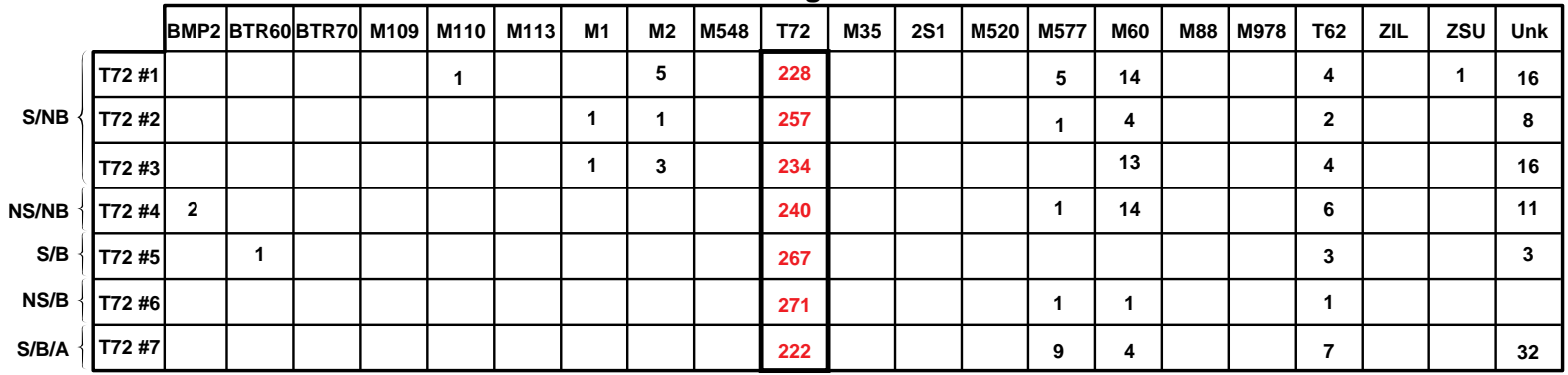

To summarize the performance of the 10- and 20-target classifier implementations described earlier, Table 6 presents confusion matrices combining the results using 5195 independent test inputs. The leftmost column denotes the target type, followed by the number of different-serial-numbered targets of each type used in the performance evaluation. For example, there were 9 different-serial-numbered T72s included in this final performance summary. Since the total number of test inputs varies with each target type, the performance numbers have been converted to percent. As Table 6 shows, the probabilities of correct classification are $95.8 \%$ and $92.6 \%$ for the 10- and 20-target classifiers, respectively. 
Table 6

Confusion Matrices for the 10- and 20-Target Classifiers (with additional T72 templates)

(Composite of MSTAR \#1 and MSTAR \#2 Data Sets)

Percent of Targets Classified As

$332022-4$

\begin{tabular}{|l|l|l|l|l|l|l|l|l|l|l|l|}
\cline { 2 - 11 } \multicolumn{1}{c|}{} & BMP2 & BTR60 & BTR70 & M109 & M110 & M113 & M1 & M2 & M548 & T72 & Unk \\
\hline BMP2 (2) & 97.0 & & 0.25 & 0.75 & & & & 0.75 & & 0.25 & 1.0 \\
\hline BTR70 (3) & & & 95.0 & & & & & & & & 5.0 \\
\hline M109 (4) & 0.1 & & & 98.0 & & & & 0.3 & & & 1.6 \\
\hline M2 (2) & & & & & & 0.25 & & 99.5 & & & 0.25 \\
\hline T72 (9) & 0.2 & 0.1 & & & 0.1 & & 0.1 & 0.5 & & 94.0 & 5.0 \\
\hline
\end{tabular}

Percent of Targets Classified As

20-Target Classifier Pcc $=92.6 \%$

\begin{tabular}{|l|l|l|l|l|l|l|l|l|l|l|l|l|l|l|l|l|l|l|l|l|l|}
\multicolumn{1}{l|}{} & BMP2 & BTR60 & BTR70 & M109 & M110 & M113 & M1 & M2 & M548 & T72 & M35 & 2S1 & M520 & M577 & M60 & M88 & M978 & T62 & ZIL & ZSU & Unk \\
\hline BMP2 (2) & 96.75 & & 0.25 & 0.25 & & & & 0.75 & & 0.25 & & & & 0.75 & & & & & & & 1.0 \\
\hline BTR70 (3) & & & 91.5 & & & & & & & & & 3.25 & & 0.25 & & 0.25 & & 0.25 & 0.5 & & 4.0 \\
\hline M109 (4) & 0.1 & & & 94.25 & & & & 0.25 & & & & & & 3.0 & & & & 0.2 & 0.7 & 0.1 & 1.4 \\
\hline M2 (2) & & & & & & 0.25 & & 99.25 & & & & & & 0.25 & & & & & & & 0.25 \\
\hline T72 (9) & 0.2 & 0.1 & & & 0.1 & & 0.2 & & & 91.0 & & & & 0.8 & 2.25 & & & 1.25 & & & 4.1 \\
\hline
\end{tabular}

\section{EXTENDED OPERATING CONDITIONS}

This section of the paper summarizes MSE classifier performance evaluations using test images of targets gathered during the MSTAR \#2 collection in various extended operating condition configurations [7]. Preliminary performance results are presented for targets that (1) are obscured by revetments, and (2) targets have significant turret rotations. For testing the effects of revetments on classifier performance, test target imagery from the MSTAR \#2 collection was used, including the BTR70, M109, and T72 targets. Figure 9 is a photograph of the BTR70 target in a half-revetment configuration; Figure 10 shows the M109 target in a full-revetment configuration; and Figure 11 shows the T72 target in a half-revetment configuration with the turret rotated 45 deg right. 


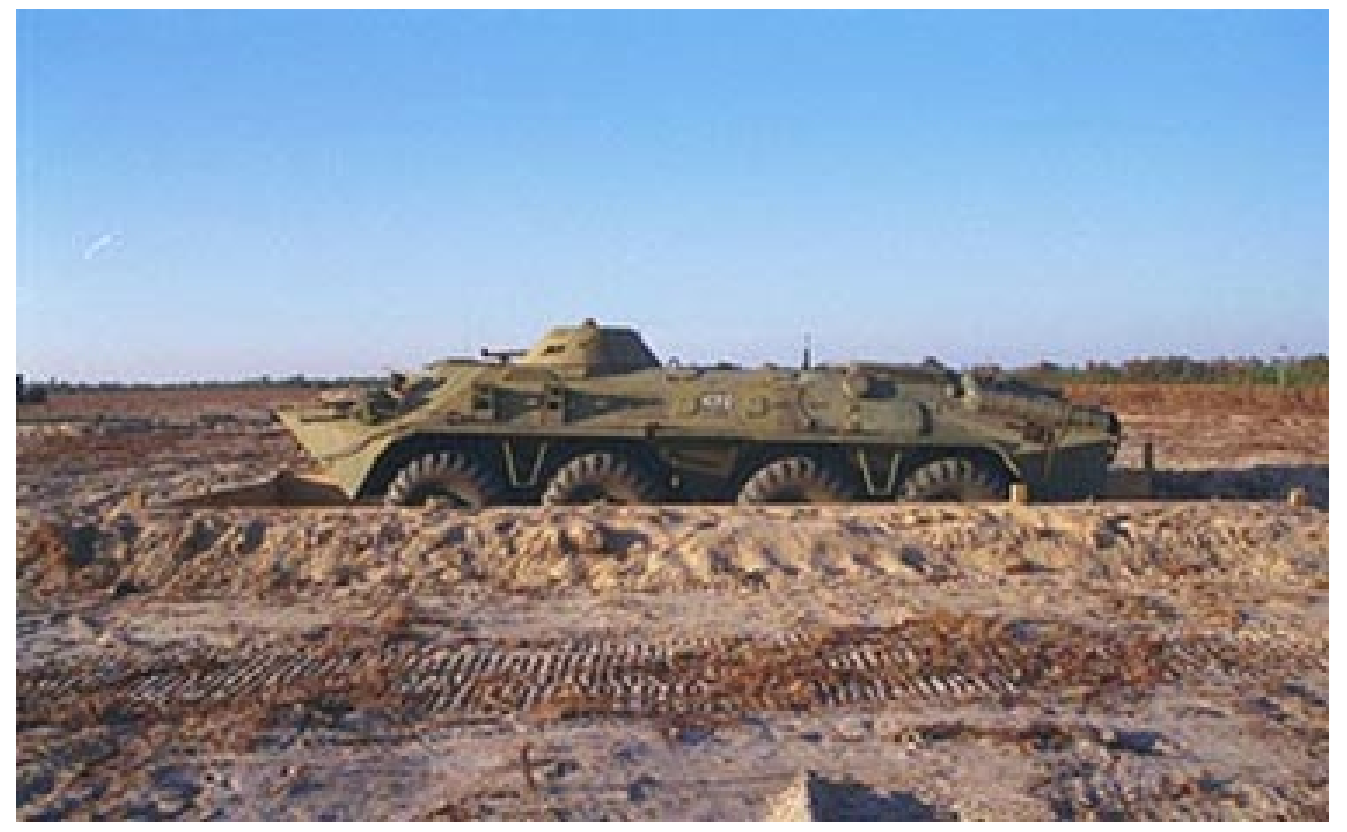

Figure 9. Photograph of a BTR70 in a half-revetment configuration. A half-revetment is designed to obscure objects below the vehicle axle.

We evaluated the MSE classifier scores for the M109 target in various revetted configurations. Figure 12 shows the MSE scores for testing against independent M109 targets (1) in the open, (2) in halfrevetments, and (3) in full-revetments (the M109 training templates were from the MSTAR \#1 data). The figure indicates that the effect of the revetments is to partially obscure the target due to blockage of the radar energy, resulting in an increase in the MSE scores. We determined that increasing the MSE threshold to 65 would provide quite good classifier performance against these revetted targets. Table 7 summarizes the results of this study for the 10-target classifier using the MSE threshold of 65; the probability of correct classifications are $50.8 \%$ and $75.7 \%$ against the fully and half-revetted targets, respectively. Using an MSE threshold of 60, the probability of correct classifications are reduced to $24.9 \%$ and $49.4 \%$ against the fully and half-revetted targets, respectively (see Table 8). Using our baseline MSE threshold of 55 (Tables 1,2,4,5 and 6) the probabilities of correct classification are further reduced to $2.2 \%$ and $13.9 \%$ against the fully and half-revetted targets (see Table 9). Although $\mathrm{P}_{\mathrm{CC}}$ against these revetted targets may be improved significantly by increasing the MSE threshold, the ability of the classifier to reject confuser vehicles would be significantly decreased. Figure 13 shows MSE classifier confuser rejection versus the MSE threshold; from these curves it is seen that the baseline 
threshold of 55 provides good confuser rejection whereas using an MSE threshold of 65 shows essentially no confuser rejection capability.

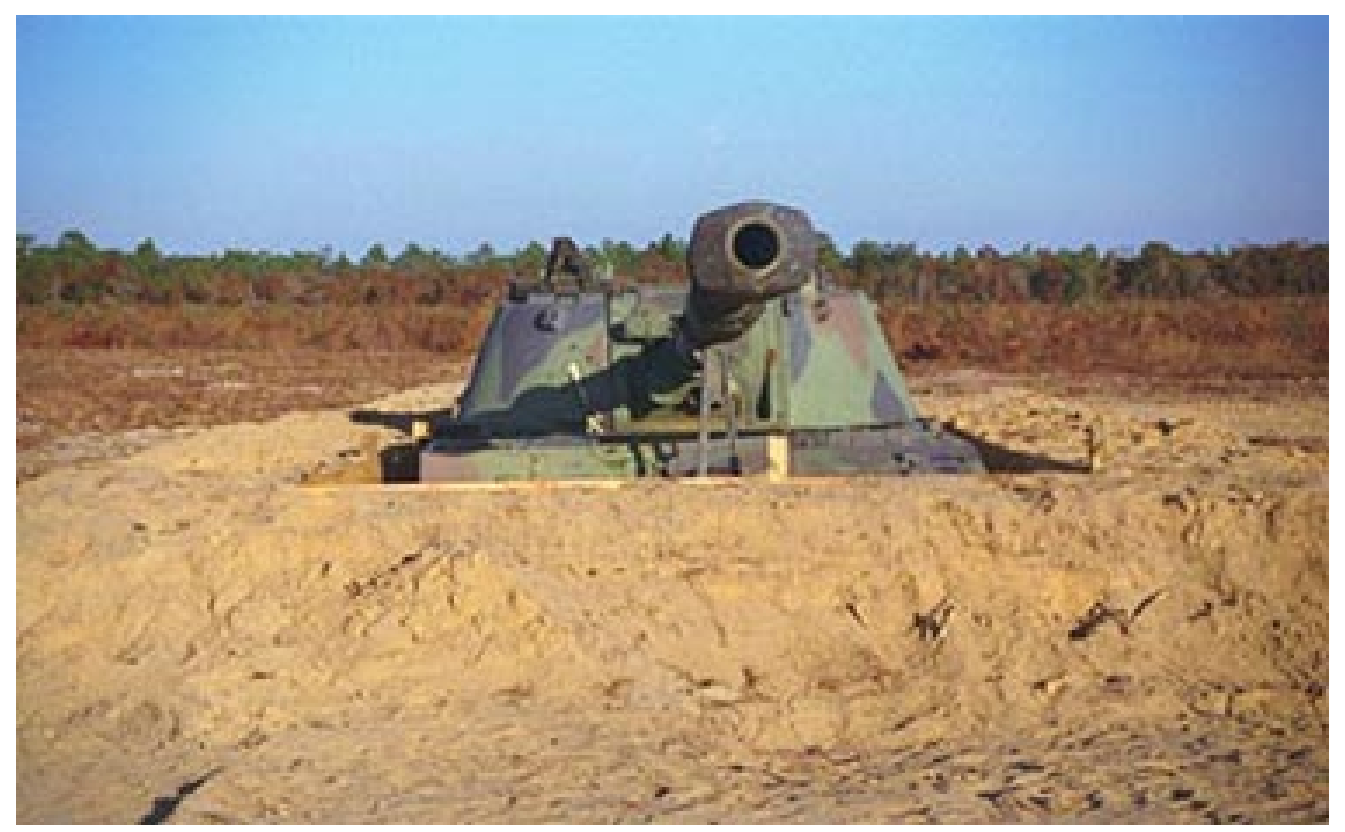

Figure 10. Photograph of an M109 in a full-revetment configuration. A full revetment is designed to obscure objects below the vehicle deck.

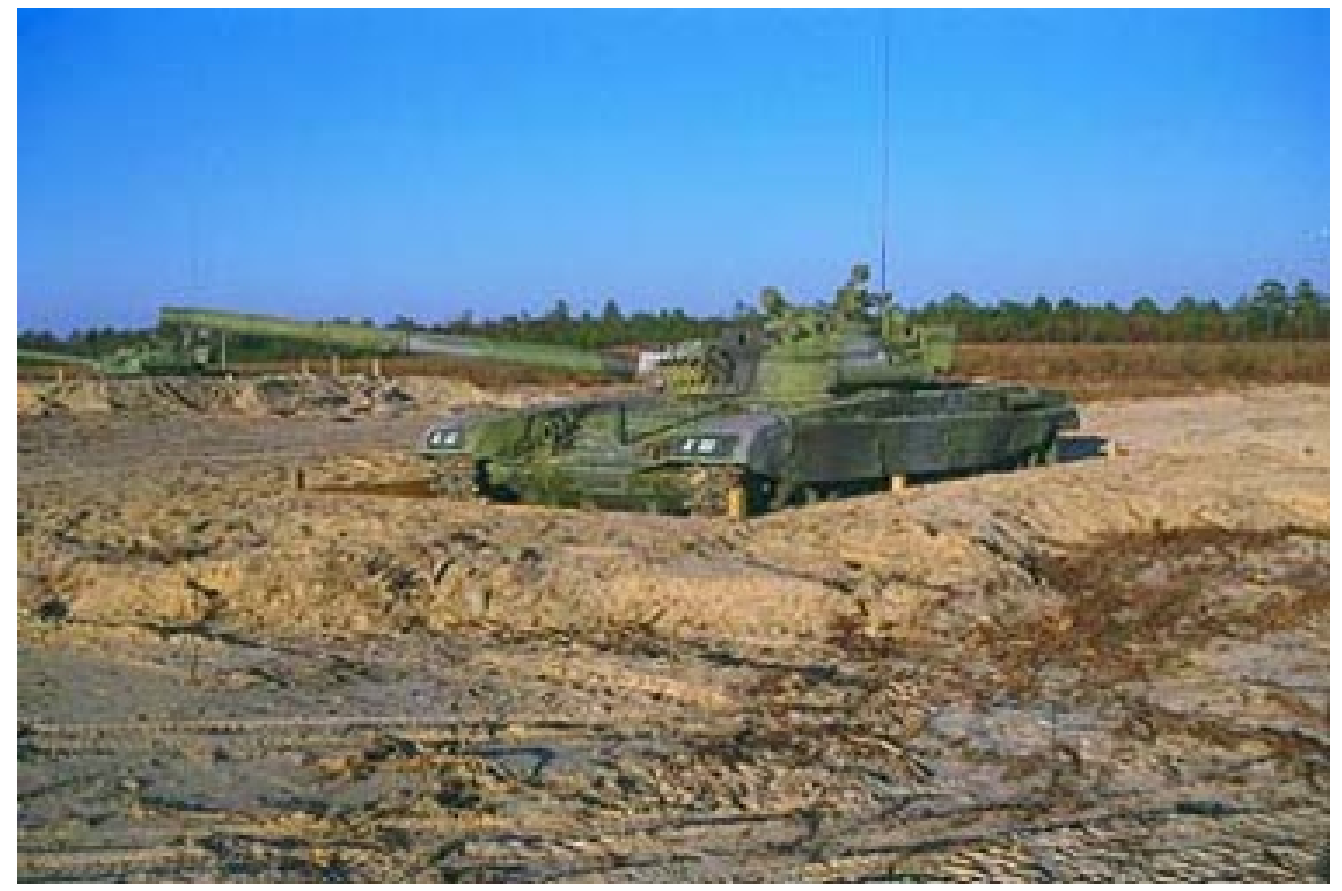

Figure 11. Photograph of a T72 in a half-revetment configuration with the turret rotated 45 deg. 


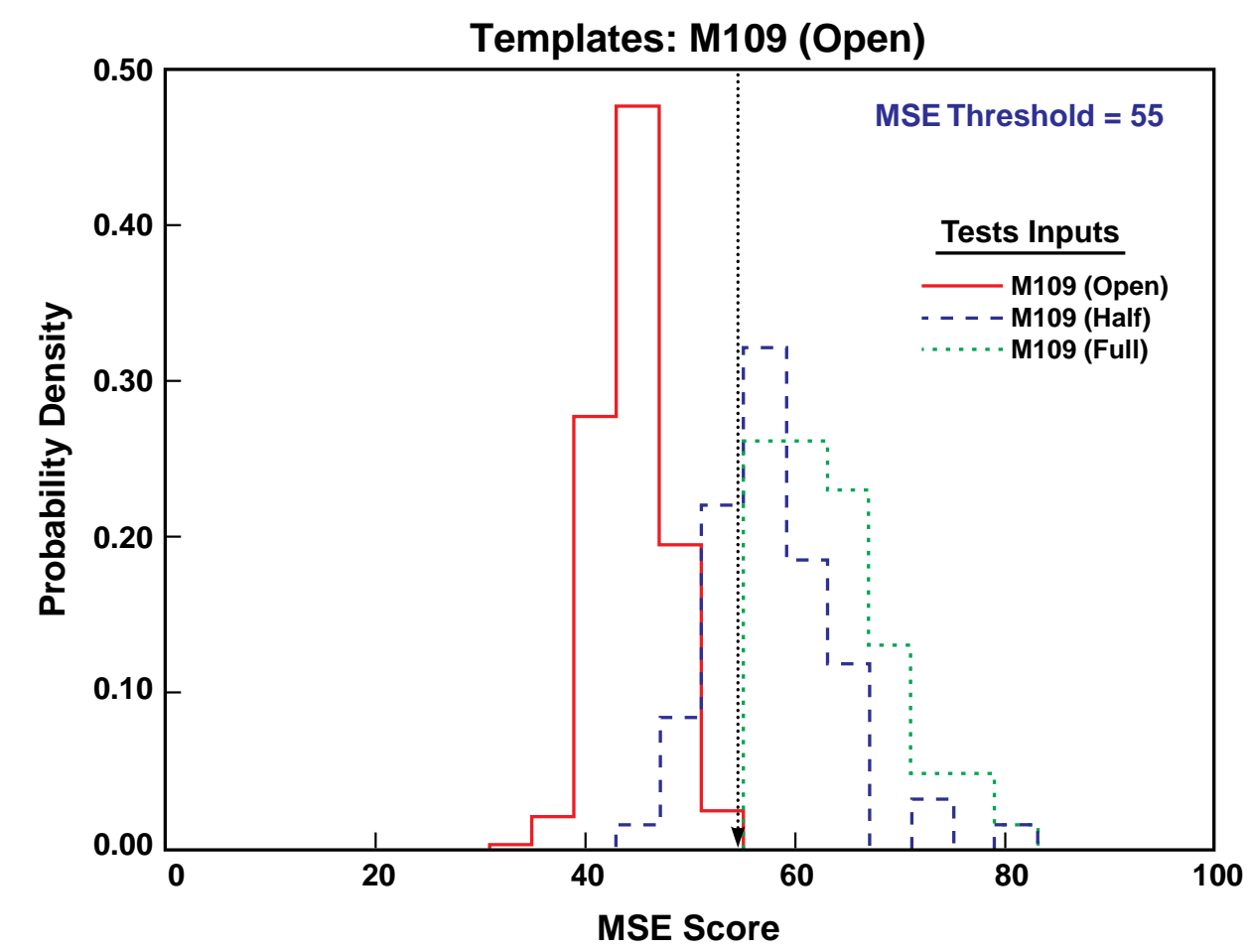

Figure 12. MSE score distributions of three independent M109 test inputs against the M109 templates used to train the classifier. The solid line is the distribution of scores from the M109 with the same configuration, in the open, as the M109 used in training. The dashed line is the distribution of scores from the M109 in the half-revetment configuration. The dotted line is the distribution of scores from the M109 in the full-revetment configuration. The MSE scores increase as the amount of obscuration of the test targets increase. 
Table 7

Confusion Matrix for the 10-Target Classifier (Threshold $=65$ )

(Six Targets From the MSTAR \#2 Data Set)

332022-2

Number of Targets Classified As

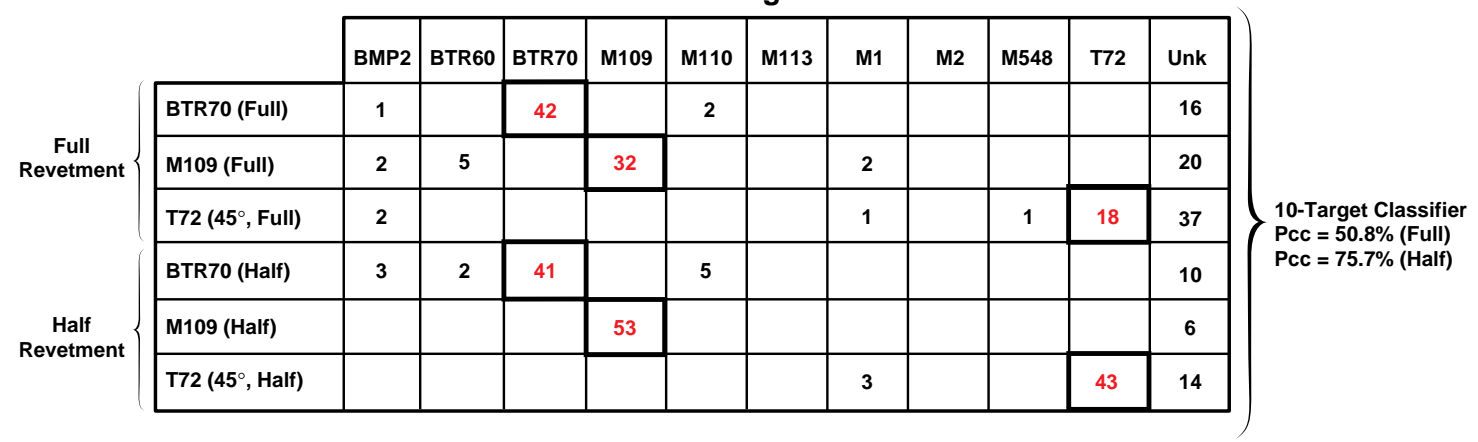

Table 8

Confusion Matrix for the 10-Target Classifier (Threshold $=60$ )

(Six Targets From the MSTAR \#2 Data Set)

Number of Targets Classified As

$332022-6$

\begin{tabular}{|c|c|c|c|c|c|c|c|c|c|c|c|c|c|}
\hline & & BMP2 & BTR60 & BTR70 & M109 & M110 & M113 & M1 & M2 & M548 & $\mathrm{T} 72$ & Unk & \multirow{7}{*}{$\begin{array}{l}\text { 10-Target Classifier } \\
\text { Pcc }=24.9 \% \text { (Full) } \\
\text { Pcc }=49.4 \% \text { (Half) }\end{array}$} \\
\hline \multirow{4}{*}{$\begin{array}{c}\text { Full } \\
\text { Revetment }\end{array}$} & BTR70 (Full) & & & 26 & & & & & & & & 35 & \\
\hline & M109 (Full) & 1 & 1 & & 16 & & & & & & & 43 & \\
\hline & T72 (45, Full $)$ & & & & & & & & & & 3 & 56 & \\
\hline & BTR70 (Half) & 2 & 2 & 24 & & 2 & & & & & & 31 & \\
\hline \multirow{2}{*}{$\begin{array}{c}\text { Half } \\
\text { Revetment }\end{array}$} & M109 (Half) & & & & 38 & & & & & & & 21 & \\
\hline & T72 $\left(45^{\circ}\right.$, Half $)$ & & & & & & & 1 & & & 27 & 32 & \\
\hline
\end{tabular}

Table 9

Confusion Matrix for the 10-Target Classifier (Threshold $=55$ )

(Six Targets From the MSTAR \#2 Data Set)

Number of Targets Classified As

\begin{tabular}{|c|c|c|c|c|c|c|c|c|c|c|c|c|c|}
\hline & & BMP2 & BTR60 E & BTR70 & M109 & M110 & M113 & M1 & M2 & M548 & $\mathrm{T72}$ & Unk & \multirow{7}{*}{$\begin{array}{l}\text { 10-Target Classifier } \\
\text { Pcc }=2.2 \% \text { (Full) } \\
\text { Pcc }=13.9 \% \text { (Half) }\end{array}$} \\
\hline Full & BTR70 (Full) & & & 4 & & & & & & & & 57 & \\
\hline \multirow[t]{3}{*}{ Revetment } & M109 (Full) & & & & 0 & & & & & & & 61 & \\
\hline & T72 (45ㅇ, Full) & & & & & & & & & & 0 & 59 & \\
\hline & BTR70 (Half) & & & 4 & & & & & & & & 57 & \\
\hline \multirow[t]{2}{*}{$\begin{array}{c}\text { Half } \\
\text { Revetment }\end{array}$} & M109 (Half) & & & & 13 & & & & & & & 46 & \\
\hline & T72 (45, Half) & & & & & & & & & & 8 & 52 & \\
\hline
\end{tabular}




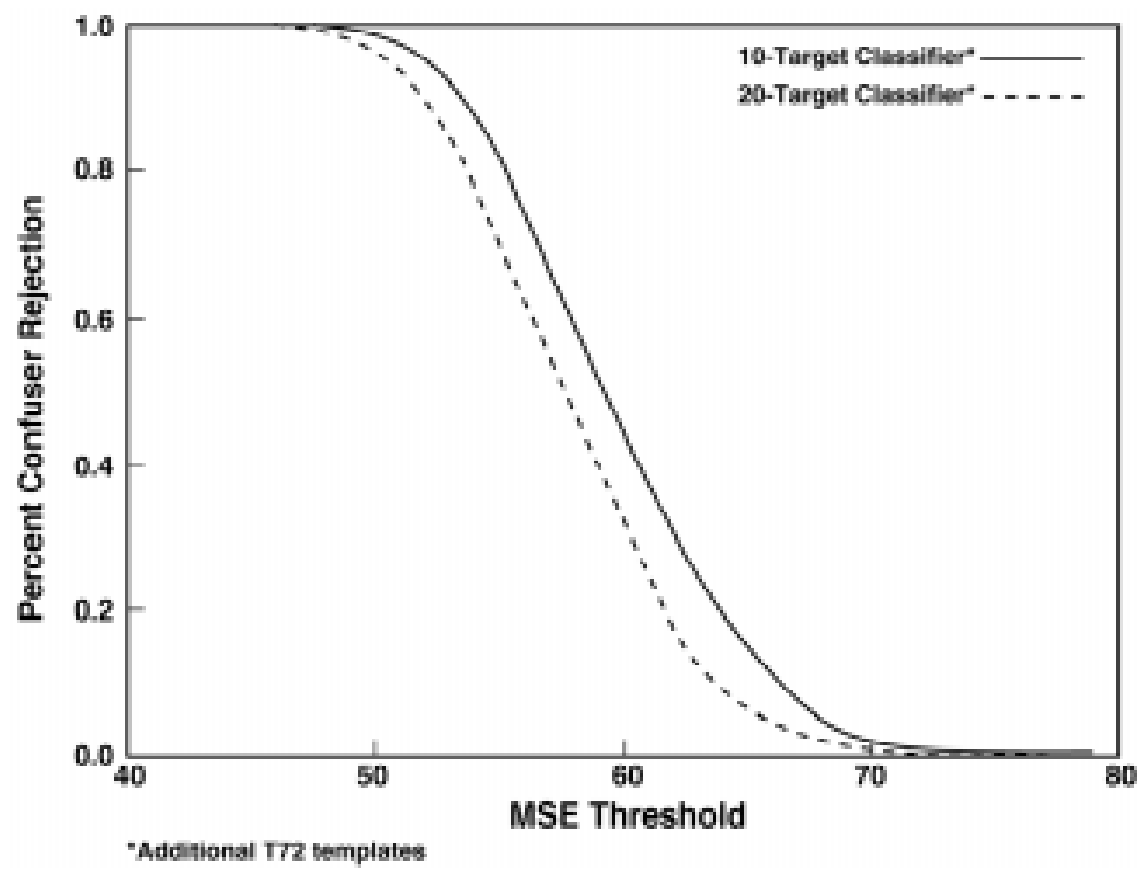

Figure 13. Probability of rejecting confuser vehicles (i.e., vehicles not included in the set of targets that the classifier was trained to recognize) versus MSE classifier threshold. A classifier threshold of 55 provides good confuser rejection.

We have investigated the effect of turret rotations for the M109 target. The turret rotations investigated were quite severe, with the maximum rotation as large as 90 deg from the nominal forward turret position (see Figure 14). The templates used in this study were constructed using the M109 target (with no turret rotation) from the MSTAR \#1 data. As Figure 15 indicates, for turret rotations of 10 deg or more, the probability of correctly classifying this target degrades very rapidly. Classifier performance could be improved by including extra templates from M109s having turret rotations at various angles; we do not have independent training data to test this hypothesis, but we speculate that it could require template sets every $20 \mathrm{deg}$ or so of turret rotation to achieve good classifier performance. 


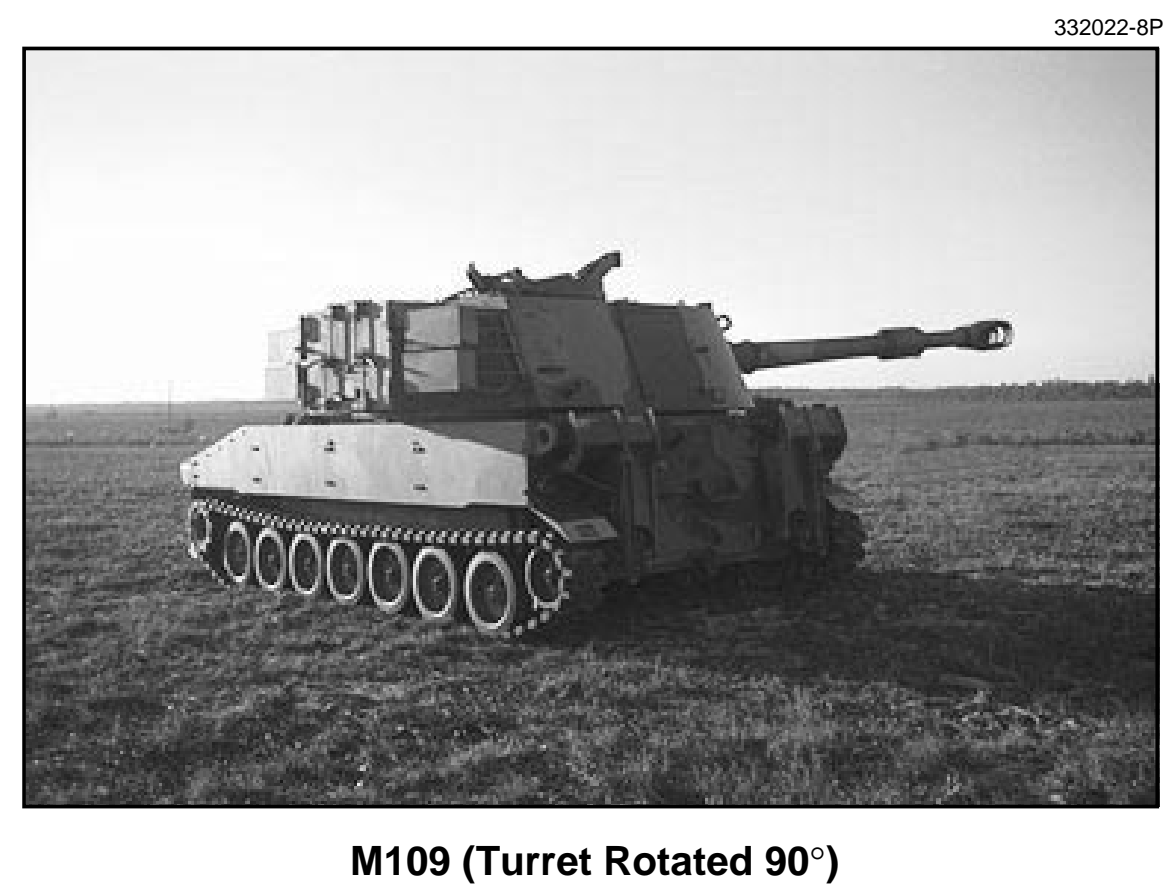

Figure 14. Photograph of an M109 with turret rotated 90 deg from forward position. 


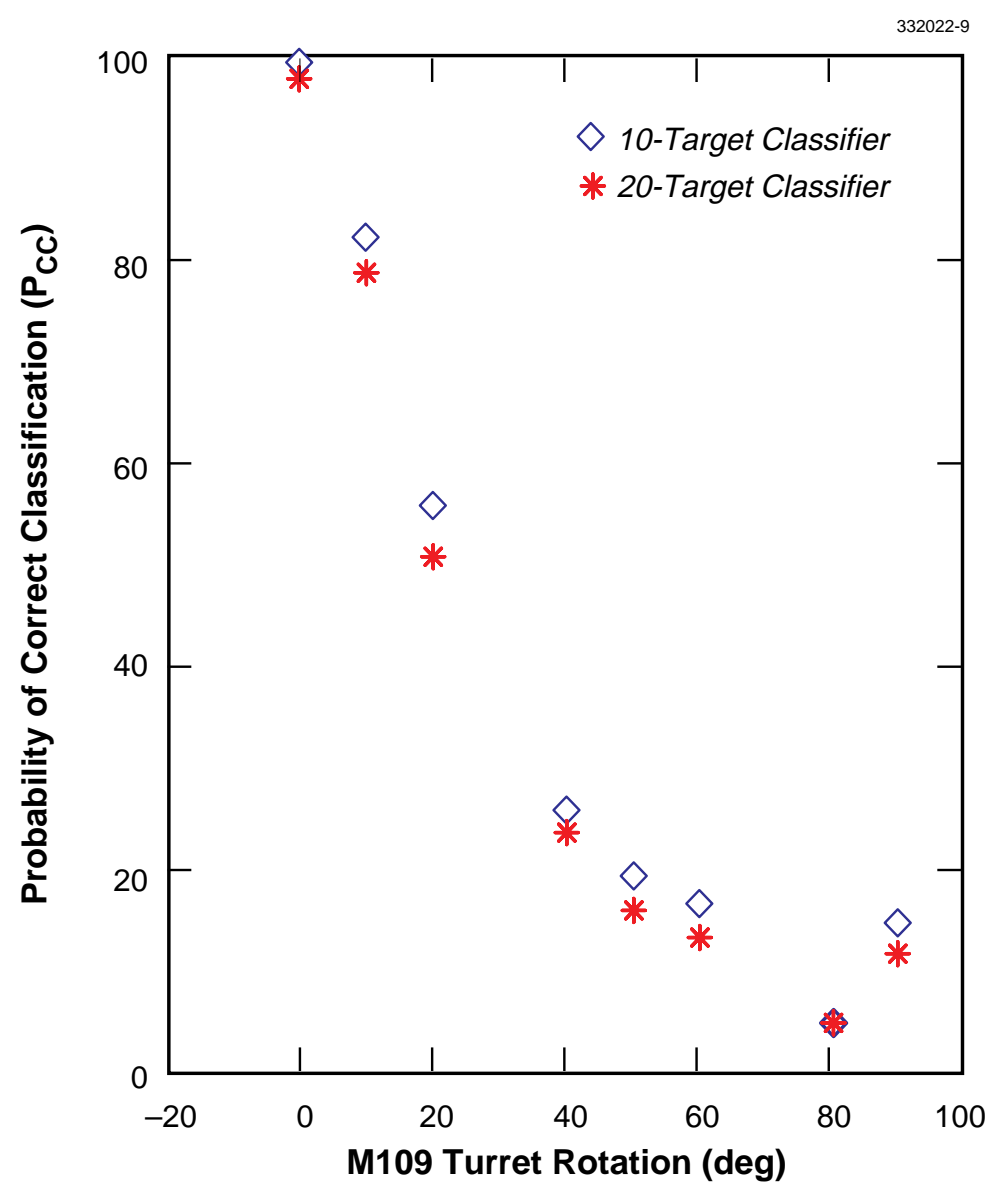

Figure 15. Probability of correct classification of an M109 in the open as a function of M109 turret rotation. The M109 used to train the 10- and 20-target classifiers was articulated with no turret rotation. The turret of the M109 represents a significant fraction of the total radar-illuminated surface. The classifier performance degrades rapidly but may be improved with the introduction of training data for M109s with rotated turrets. 


\section{SUMMARY}

This paper has presented an evaluation of the performance of 10-target and 20-target, template-based MSE classifiers; both classifiers were developed at Lincoln Laboratory in support of the SAIP program. Classifier performance was evaluated using a large number of images of tactical military targets (5195 test images) at high resolution. The results of these performance evaluations show that the number of target classes can be increased from 10-targets to 20-targets with only a small decrease in target recognition performance. The correct classification performance for the final 10- and 20-target classifiers was $95.8 \%$ and $92.6 \%$, respectively. The results of these evaluations show that significant target configuration variability can decrease interclass separability and degrade performance; however, additional reference templates can be used to mitigate these effects. Finally, these evaluations also show that classifier performance degrades significantly when tested against targets in revetments; this is because the revetments obscure significant portions of the target's signature.

\section{REFERENCES}

1. L.M. Novak, G.J. Owirka, W.S. Brower, and A.L. Weaver, "The Automatic Target Recognition System in SAIP," Lincoln Laboratory Journal, Vol. 10, No. 2, 1997.

2. L.M. Novak, G.J. Owirka, and A.L. Weaver, "Automatic Target Recognition Using Enhanced Resolution SAR Data," IEEE Transactions on Aerospace and Electronic Systems, Vol. 35, No. 1, January 1999.

3. W.P. Delaney, "The Changing World, The Changing Nature of Conflicts: A Critical Role for Military Radar," 1995 IEEE National Radar Conference, Alexandria, VA, May 1995.

4. Global Hawk Relays Images Via Commercial Satellite," Aviation Week and Space Technology, 15 February 1999.

5. L.M. Novak, G.J. Owirka, and W.S. Brower, "An Efficient Multi-Target SAR ATR Algorithm," Asilomar Conference, Pacific Grove, CA, November 1998.

6. Moving and Stationary target Acquisition Recognition (MSTAR), Program Technology Review, Denver, CO, November 1996.

6. E.R. Keydel, and S.W. Lee, "MSTAR Extended Operating Conditions: A Tutorial," SPIE 2757, Orlando, FL, April 1996. 